УДК $005.344: 629.7 .01$

О. Е. ФЕДОРОВИЧ ${ }^{1}$, О. А. ГАЙДЕНКО ${ }^{1}$, В. А. ПУЙДЕНКО 2

${ }^{1}$ Национальный аэрокосмический университет им. Н. Е. Жуковского «ХАИ», Украина

${ }^{2}$ Харьковский радиотехнический техникум, Украина

\title{
ИСПОЛЬЗОВАНИЕ КОМПОНЕНТНО-КОМПЕТЕНТНОСТНОГО ПОДХОДА ДЛЯ ФОРМИРОВАНИЯ КООПЕРАЦИИ ИСПОЛНИТЕЛЕЙ ИННОВАЦИОННОГО ЗАКАЗА ПРИ СОЗДАНИИ СЛОЖНОЙ АЭРОКОСМИЧЕСКОЙ ТЕХНИКИ
}

\begin{abstract}
Поставлена и решена задача создания кооперации исполнителей инновачионного заказа по созданию новой аэрокосмической техники (АКТ) на основе компонентно-компетентностного подхода. Для этого на начальных этапах проектирования архитектура будущего изделия АКТ представлена в виде компонент (инновационных и из прошлого опыта разработок). Каждая создаваемая компонента оценивается с точки зрения компетентности, в виде составляющих-компетенций (знания, умения, навыки, опьтт). Для этого, с помощью экспертов, формируются требования по компетентности для каждой из компонент изделия. Для поиска ответных компетентностей у возможных кандидатов в исполнители проекта создана база прецедентов (БП), в которую вносятся оценки независимых экспертов по отдельным компетенциям кандидатов. Далее сравниваются требования заказчика, оформленные в виде контрольного «слова» с компетенциями кандидатов в БП по каждой компоненте сложного изделия. Использованы количественные и качественные представления экспертами оценок компетенций с использованием методов целочисленного линейного программирования и лексикографического упорядочивания вариантов. Предложенный подход иелесообразно использовать на начальных этапах инновацчинного проекта, когда необходимо создать кооперациюю исполнителей и при этом учитьвать выполнение требований по компетентности со стороны заказчика, основанные на анализе компонентной архитектуры сложного изделия, в которой выделены компоненты прошлых разработок и новые инновачионные компоненты.
\end{abstract}

Ключевые слова: метод компонентного проектирования, компетентностный подход, компетенция, инновационный проект, опыт прошлых разработок, база прецедентов, лексикографическое упорядочивание вариантов.

\section{Введение}

Устойчивое поведение производственной системы в условиях конкуренции и изменений запросов потребителей на рынке высокотехнологической продукции (авиастроение, судостроение и т.д.) требует поиска новых форм организации и управления производством. Одной из прогрессивных форм организации является виртуальная производственная система, кооперация в которой создается на период выполнения актуального заказа, что позволяет оперативно реагировать на запросы рынка [1].

При выполнении инновационного заказа по созданию новой аэрокосмической техники (АКТ), необходимо сформировать кооперацию исполнителей, у которых есть необходимый опыт, знания и умения, соответствующие требованиям технического задания инновационного проекта [2].

Совместное использование компетентностного подхода для выбора исполнителей и компонентной архитектуры инновационного изделия позволяет рационально подойти к формированию кооперации для участия в проекте [3].

Поэтому актуальна тема предлагаемой публикации, в которой ставится и решается задача формирования кооперации исполнителей виртуальной производственной системы с использованием компонентно-компетентностного подхода.

\section{Постановка задачи исследования}

На начальном этапе создания наукоемкого изделия формируется компонентная архитектура сложного изделия, в которой компоненты имеют разную степень новизны (инновационности). Можно выделить три типа возможных компонент инновационного изделия АКТ:

- инновационные компоненты (ИК);

- компоненты прошлых разработок (КПР), которые, возможно, нуждаются в адаптации в рассматриваемом проекте;

- сложные компоненты, включающие ИК и КПР.

(c) О. Е. Федорович, О. А. Гайденко, В. А. Пуйденко 
Позитивный опыт прошлого позволяет минимизировать риск, связанный с созданием компонент и обеспечивает сокращение затрат и сроков разработки и производства новых изделий. Инновационные компоненты с одной стороны увеличивают риск, затраты и сроки их реализации, а с другой могут обеспечить конкурентоспособность изделия на рынке наукоемкой техники. Поэтому, при создании изделий типа АКТ, необходимо найти компромисс между инновационностью, конкурентоспособностью, рисками и затратами.

Компетентностный подход позволяет оценить каждую проектируемую компоненту с точки зрения требований уровня компетентности от исполнителей (знаний, умений, навыков и опыта) для её создания. Для выбора подходящего состава исполнителей для выполнения работ по созданию компонент необходимо сравнить возможности кандидатов исполнителей по уровням их компетентности. При этом необходимо учитывать специфику знаний, умений и навыков, требуемых для реализации данной компоненты, чтобы найти исполнителя с требуемым уровнем компетентности.

Представим критерии для оценки компетентности в виде вектора $\overrightarrow{\mathrm{K}}$, в который входят два вида составляющих:

1) составляющие, связанные непосредственно с оценкой компетентности:

- уровень знаний, необходимый для выполнения заказа (отдельной компоненты изделия) - YZ;

- уровень умений, необходимых для выполнения заказа (компоненты изделия) - YY ;

- уровень практических навыков, необходимых для создания инновационного изделия (компоненты изделия) - YN ;

- наличие опыта по созданию близких по своим характеристикам и требованиям изделий (компонент изделий) - ҮР ;

2) составляющие, связанные с обеспечением необходимых требований по компетентности:

- затраты исполнителей заказа, связанные с обеспечением необходимых требований по компетентности (переподготовка кадров, набор новых сотрудников и т.д.) - ZL;

- время, которое необходимо затратить, чтобы обеспечить требуемый уровень компетентности для выполнения заказа - ТK ;

- риск, связанный с включением исполнителя в кооперацию для выполнения инновационного заказа $-\mathrm{RK}$.

Необходимо с учетом требований по компетентности для каждой i-й компоненты заказа $\mathrm{i}=\overline{1, \mathrm{n}}$, связанного с созданием сложного изделия АКТ, по- добрать исполнителей для формирования кооперации по выполнению инновационного проекта.

\section{Решение задачи исследования}

Для выбора исполнителей создаваемого инновационного изделия необходимо сформировать информационную базу с характеристиками возможных кандидатов.

Представим кандидатов в исполнители заказа в виде прецедентов. Тогда, поиск требуемых исполнителей и включение их в состав кооперации будет осуществляться с помощью базы прецедентов (БП), где каждый ј-й возможный исполнитель по созданию і-й компоненты может быть представлен в виде составляющих вектора $\overrightarrow{\mathrm{K}}$ :

$$
\overrightarrow{\mathrm{K}}_{\mathrm{ij}}=\left\{\mathrm{YZ}_{\mathrm{ij}}, \mathrm{YY}_{\mathrm{ij}}, \mathrm{YN}_{\mathrm{ij}}, \mathrm{YP}_{\mathrm{ij}}, \mathrm{ZL}_{\mathrm{ij}}, \mathrm{TK}_{\mathrm{ij}}, \mathrm{RK}_{\mathrm{ij}}\right\} .
$$

Необходимо для каждой і-й компоненты сложного изделия подобрать такого ј-го исполнителя, для которого $\overrightarrow{\mathrm{K}}_{\mathrm{ij}}$ будет наилучшим в некотором смысле (например, близость компетенций требуемых для создания і-й компоненты инновационного заказа и компетенций ј-го исполнителя).

Для оценки требуемой компетентности в заказе и компетентности исполнителей можно воспользоваться количественными и качественными оценками:

1. Формирование кооперации исполнителей инновационного заказа с использованием количественных оценок компетентности.

В этом случае каждую составляющую компетентности как для заказа так и исполнителей, можно представить в некоторой нормированной шкале (например $0 \div 10$ ). Будем считать, что для выполнения заказа $Z$ значения оценок, связанных с компетентностью должны приближаться к максимальным значениям $\quad(\mathrm{YZ}=10, \mathrm{YY}=10, \mathrm{YN}=10, \mathrm{YP}=10)$. Поэтому необходимо выбрать из БП такие прецеденты (кандидаты в исполнители), у которых будут наилучшие значения уровней знаний, умений, навыков и опыта. Введем комплексную оценку компетентности в виде:

$$
\mathrm{Q}=\alpha_{\mathrm{Z}} \cdot \mathrm{YZ}+\alpha_{\mathrm{Y}} \cdot \mathrm{YY}+\alpha_{\mathrm{N}} \cdot \mathrm{YN}+\alpha_{\mathrm{P}} \cdot \mathrm{YP},
$$

где $\alpha_{\mathrm{q}}$ - весовые оценки компетенций (знаний, умений, навыков, опыта), $0 \leq \alpha_{\mathrm{q}} \leq 1, \sum_{\mathrm{q}=1}^{4} \alpha_{\mathrm{q}}=1$, зна- 
чение $\alpha_{\mathrm{q}}$ задают представители заказчика инновационного проекта.

Для выбора состава кооперации исполнителей инновационного заказа воспользуемся методом целочисленного линейного программирования. Необходимо максимизировать значение Q :

$$
\begin{gathered}
\max Q, Q=\alpha_{Z} \cdot \sum_{i=1}^{n} \sum_{j=1}^{n_{i}} Y Z_{i j}+\alpha_{Y} \cdot \sum_{i=1}^{n} \sum_{j=1}^{n_{i}} Y Y_{i j}+ \\
+\alpha_{N} \cdot \sum_{i=1}^{n} \sum_{j=1}^{n_{i}} Y N_{i j}+\alpha_{P} \cdot \sum_{i=1}^{n} \sum_{j=1}^{n_{i}} Y P_{i j}
\end{gathered}
$$

при выполнении заданных ограничений на затраты, время и риски:

$$
\begin{gathered}
Z L \leq Z^{\prime}, Z L=\sum_{i=1}^{n} \sum_{j=1}^{n_{i}} Z_{i j} ; \\
T K \leq T K^{\prime}, T K=\sum_{i=1}^{n} \sum_{j=1}^{n_{i}} T_{i j} ; \\
R K \leq R K^{\prime}, R K=\sum_{i=1}^{n} \sum_{j=1}^{n_{i}} R K_{i j},
\end{gathered}
$$

где ZL', TK', RK' - допустимые затраты, время и риски.

Поиск наилучших вариантов состава исполнителей будем осуществлять в созданной базе прецедентов, в которой хранятся характеристики компетентностей для возможных кандидатов в исполнители по каждой і-й компоненте сложного инновационного изделия.

2. Формирование кооперации исполнителей инновационного заказа с использованием качественных оценок компетентности.

Качественные оценки удобно использовать на начальных этапах выполнения инновационного проекта, когда из-за возможной неопределенности, трудно количественно оценить не только компетентность, но и характеристики будущего изделия. Особенно это характерно для этапов научных исследований и опытно-конструкторских работ, где основным требованием к исполнителям является наличие требуемого уровня компетентности. В качестве качественной шкалы используем буквы латинского алфавита, которые будут представлять значения оценок компетенций. В результате получим:

$$
\mathrm{YZ}=\left\{\begin{array}{l}
\text { A - высокий уровень знаний, } \\
\text { В - достаточный уровень знаний, } \\
\text { C- удовлетворительный уровень знаний, } \\
\mathrm{D}-\text { низкий уровень знаний; }
\end{array}\right.
$$

$\mathrm{YY}=\left\{\begin{array}{l}\text { A - высокий уровень умений, } \\ \text { В-достаточный уровень умений, } \\ \text { C- удовлетворительный уровень умений, } \\ \text { D- низкий уровень умений; }\end{array}\right.$

$\mathrm{YN}=\left\{\begin{array}{c}\text { A - высокий уровень практических навыков, } \\ \mathrm{B}-\text { достаточный уровень } \\ \quad \text { практических навыков, } \\ \mathrm{C}-\text { удовлетворительный уровень } \\ \quad \text { практических навыков, } \\ \mathrm{D}-\text { низкий уровень практических навыков; }\end{array}\right.$

$\mathrm{YP}=\left\{\begin{array}{l}\text { A - высокий уровень опыта, } \\ \text { В-достаточный уровень опыта, } \\ \mathrm{C}-\text { удовлетворительный уровень опыта, } \\ \mathrm{D}-\text { низкий уровень опыта; }\end{array}\right.$

$\mathrm{ZL}=\left\{\begin{array}{l}\text { A - минимальные затраты, } \\ \text { B- удовлетворительные затраты, } \\ \mathrm{C}-\text { большие затраты, } \\ \mathrm{D}-\text { очень большие затраты; }\end{array}\right.$

$\mathrm{TK}=\left\{\begin{array}{l}\text { A - минимальное время, } \\ \text { B - удовлетворительное время, } \\ \mathrm{C}-\text { большие временные затраты, } \\ \mathrm{D}-\text { очень большие временные затраты; }\end{array}\right.$

$\mathrm{RK}=\left\{\begin{array}{l}\text { A - минимальный риск, } \\ \mathrm{B}-\text { удовлетворительный риск, } \\ \mathrm{C}-\text { большой риск, } \\ \mathrm{D}-\text { очень большой риск. }\end{array}\right.$

Для оценки компетентности с помощью качественных представлений составляющих (компетенций) необходимо, вначале, проранжировать по важности компетенции в векторе $\overrightarrow{\mathrm{K}}$. С помощью экспертных мнений проведем ранжирование компетенций (основные и вспомогательные составляющие) в порядке убывания важности, т.е. установим линейный порядок. Например, пусть:

$$
\mathrm{YP} \prec \mathrm{YN} \prec \mathrm{YY} \prec \mathrm{YZ} \prec \mathrm{ZL} \prec \mathrm{TK} \prec \mathrm{RK},
$$

где $\prec-$ знак отношения предпочтения.

Далее, необходимо провести качественную оценку компетенций для всех кандидатов (прецедентов в базе прецедентов) в исполнители заказа по рассматриваемой компоненте и расположить оценки, для каждой компетенции прецедента, в соответствии с установленным порядком важности оценок.

Полученный список прецедентов в БП в виде так называемых «слов» (качественных оценок компетенций) необходимо лексикографически упорядочить для сравнения со значениями требований ком- 
петентности со стороны заказчика. Для этого заказчик задает, так называемое, контрольное «слово», которое сравнивается со «словами» прецедентов в сформированном упорядоченном списке.

Рассмотрим иллюстрированный пример. Пусть, по рассматриваемой компоненте заказа имеется следующее неупорядоченное множество прецедентов:

$$
\begin{aligned}
& \text { 1. B, A, C, B, B, B, D; } \\
& \text { 2. A, C, B, A, C, B, D; } \\
& \text { 3. B, B, A, B, C, B, B; } \\
& \text { 4. A, B, C, B, C, B, B. }
\end{aligned}
$$

После лексикографического упорядочивания получим:

$$
\begin{aligned}
& \text { 4. A, B, C, B, C, B, B; } \\
& \text { 2. A, C, B, A, C, B, D; } \\
& \text { 1. B, A, C, B, B, B, D; } \\
& \text { 3. B, B, A, B, C, B, B. }
\end{aligned}
$$

Возможны следующие ситуации, связанные с расположением контрольного «слова» $\mathrm{S}$ компетентности со стороны заказчика в упорядоченном списке кандидатов (прецедентов):

1. Контрольное «слово» $\mathrm{S}$ расположено выше упорядоченного списка прецедентов:

$$
\begin{aligned}
& \text { S. A, A, B, A, B, B, A; } \\
& \text { 4. A, B, C, B, C, B, B; } \\
& \text { 2. A, C, B, A, C, B, D; } \\
& \text { 1. B, A, C, B, B, B, D; } \\
& \text { 3. B, B, A, B, C, B, B. }
\end{aligned}
$$

Это означает, что ни один кандидат в исполнители заказа не соответствует требованиям заказчика (отсутствует требуемая компетентность исполнителей с учетом требований заказчика). В этом случае, необходимо привлечь новых кандидатов в исполнители. Если это не удается, то необходимо снизить требования компетентности к исполнителям.

2. Контрольное «слово» $\mathrm{S}$ расположено в конце упорядоченного списка прецедентов:

$$
\begin{aligned}
& \text { 4. A, B, C, B, C, B, B; } \\
& \text { 2. A, C, B, A, C, B, D; } \\
& \text { 1. B, A, C, B, B, B, D; } \\
& \text { 3. B, B, A, B, C, B, B; } \\
& \text { S. B, B, B, B, B, B, A. }
\end{aligned}
$$

В этом случае любой вариант из списка прецедентов может быть использован в качестве исполнителя заказа. Целесообразно выбрать варианты из вершины списка. Например, 4 или 2.

3. Контрольное «слово» $\mathrm{S}$ расположено внутри упорядоченного списка прецедентов:
4. A, B, C, B, C, B, B;
S. A, B, C, B, C, B, C;
2. A, C, B, A, C, B, D;
1. B, A, C, B, B, B, D;
3. B, B, A, B, C, B, B.

В этом случае лучшим кандидатом в исполнители инновационного заказа будет 4-й вариант, так как его характеристики компетентности не хуже, чем требования заказчика.

\section{Выводы}

Предложенный компонентно-компетентностный подход позволяет на начальных этапах выполнения инновационного проекта осуществлять рациональный выбор исполнителей для формирования кооперации по созданию наукоемкого изделия. Компонентное представление изделия, степень новизны отдельных компонент, позволяют определить требуемые компетенции заказа для поиска в базе прецедентов ответных компетенций у кандидатов в исполнители проекта.

\section{Литература}

1. Логистика инновационной деятельности [Электронный ресурс]. - Режим доступа: htpp://rusportal.net/articles/publikacii-avtorov/ nauchnyeissledovanija/detail/5826/. - 15.02.2016

2. Исакова, Н. Ю. Интерактивная парадигма объединения предприятий в условиях глобализачии экономики и интеграции капитала [Текст] / Н. Ю. Исакова // Фундаментальные исследования. - 2013. - № 10. - C. $163-167$.

3. Федорович, О. Е. Компонентное проектирование аэрокосмической техники: моногр. [Текст] / О. Е. Федорович, Е. С. Яиина, И. В. Белецикии. -Х. : Нац. аэрокосм. ун-т «Харьк. авиаи. ин-т», 2012. $-180 \mathrm{c}$.

\section{References}

1. Logistika innovatsionnoy deyatel'nosti: elektronnyy resurs. [Logistics of innovation activity. Electronic resource]. Available at: htpp://rusportal.net/ articles/publikacii-avtorov/nauchnyeissledovanija/ detail/5826/ (accessed 15.02.2016).

2. Isakova, N. Yu. Interaktivnaya paradigma $o b$ "yedineniya predpriyatiy $v$ usloviyakh globalizatsii ekonomiki $i$ integratsii kapitala [Interactive paradigm of uniting enterprises in the context of globalization of the economy and the integration of capital]. Fundamental'nyye issledovaniya, 2013, no. 10, pp. 163 $-167$.

3. Fedorovich, O. Ye., Yashina, Ye. S., Beletskiy I. V. Komponentnoye proyektirovaniye aerokosmicheskoy tekhniki [Component design of aerospace engineering]. Kharkov, Nac. ajerokosm. un-t «Har'k. aviac. in-t» Publ., 2012. 180 p. 


\section{ВИКОРИСТАННЯ КОМПОНЕНТНО-КОМПЕТЕНТНІСНОГО ПІДХОДУ ДЛЯ ФОРМУВАННЯ КООПЕРАЦІЇ ВИКОНАВЦІВ ІННОВАЦЙНОГО ЗАМОВЛЕННЯ ДЛЯ СТВОРЕННЯ СКЛАДНОЇ АЕРОКОСМІЧНОЇ ТЕХНІКИ}

\section{О. С. Федорович, О. О. Гайденко, В.О. Пуйденко}

Поставлено та розв'язано задачу створення кооперації виконавців інноваційного замовлення по створенню нової аерокосмічної техніки (АКТ) на основі компонентно-компетентнісного підходу. Для цього на початкових етапах проектування архітектура майбутнього виробу АКТ представлена у вигляді компонент (інноваційних і з минулого досвіду розробок). Кожна створювана компонента оцінюється, з точки зору компетентності, у вигляді складових-компетенцій (знання, вміння, навички, досвід). Для цього за допомогою експертів, формуються вимоги по компетентності для кожної з компонент виробу. Для пошуку відповідних компетентностей у можливих кандидатів у виконавці проекту створено базу прецедентів (БП), в яку вносяться оцінки незалежних експертів з окремих компетенцій кандидатів. Далі порівнюються вимоги замовника, які оформляються у вигляді контрольного «слова» 3 компетенціями кандидатів в БП по кожній компоненті складного виробу. Використано кількісні і якісні уявлення експертами оцінок компетенцій із використанням методів цілочисельного лінійного програмування і лексикографічного упорядкування варіантів. Запропонований підхід доцільно використовувати на початкових етапах інноваційного проекту, коли необхідно створити кооперацію виконавців і при цьому враховувати виконання вимог щодо компетентності 3 боку замовника, які засновано на аналізі компонентної архітектури складного виробу, в якій виділено компоненти минулих розробок і нові інноваційні компоненти.

Ключові слова: метод компонентного проектування, компетентнісний підхід, компетенція, інноваційний проект, досвід минулих розробок, база прецедентів, лексикографічне упорядкування варіантів.

\section{USE OF COMPONENT-COMPETENT APPROACH FOR COOPERATION OF EXECUTERS OF INNOVATIVE ORDER DURING THE CREATION OF COMPLEX AEROSPACE TECHNIQUE}

\section{O. Ye. Fedorovich, O. A. Gaydenko, V. A. Puydenko}

The task of creation of innovative order executors' cooperation to create the new aerospace technique (AST) on the basis of the component-competence approach has been set and solved. In order to do so at the initial design stages the architecture of future AST article is represented as a component (both innovative and from the past development experience). Each created component is evaluated in terms of competence, in the form of competence components (knowledge, skills, attainments, experience). For that to happen with the help of experts competence requirements are formed for each component of the article. In order to find the responsive competencies among the potential candidates to implement the project precedent base (PB) has been created. PB contains the assessments of independent experts on specific competencies of the candidates. Further, the customer's requirements, formalized in the form of a control "word" are compared with the competencies of candidates in PB for each component of a complex article. Quantitative and qualitative expert assessments of competencies based on quantitative linear programming methods and lexicographic ordering of alternatives were used. The proposed approach is advisable to be used at the initial stages of the innovation project, when it is necessary to create the executors' cooperation and, at the same time, to consider the fulfillment of the requirements for the competence on the customer side, that are based on the analysis of the component architecture of a complex article, in which both the components of past development and new, innovative components are highlighted.

Keywords: method of component based design, competence approach, competence, innovative project, experience of past development, precedent base, lexicographic ordering of alternatives.

Федорович Олег Евгеньевич - д-р техн. наук, проф., зав. каф. информационных управляющих систем, Национальный аэрокосмический университет им. Н.Е. Жуковского «Харьковский авиационный институт», Харьков, Украина, e-mail: o.fedarovich@khai.edu.

Гайденко Олег Александрович - аспирант каф. информационных управляющих систем, Национальный аэрокосмический университет им. Н. Е. Жуковского «Харьковский авиационный институт», Харьков, Украина, e-mail: o.gaydenko@khai.edu.

Пуйденко Вадим Алексеевич - преподаватель компьютерных дисциплин, специалист высшей категории, Харьковский радиотехнический техникум, Харьков, Украина, e-mail: vapuydenko@gmail.com.

Fedorovich Oleg Yevgenyevich - Doctor of Science on Engineering, Professor, Head of the Department of Information Control Systems, National Aerospace University «Kharkov Aviation Institute», Kharkov, Ukraine, e-mail: o.fedorovich@khai.edu.

Gaidenko Oleg Aleksandrovitch - PhD student, Department of Information Control Systems, National Aerospace University «Kharkov Aviation Institute», Kharkov, Ukraine, e-mail: o.gaidenko@khai.edu.

Puydenko Vadim Alekseevich - teacher of computer disciplines, expert of the highest category, Kharkov radio technical school, Kharkiv, Ukraine, e-mail: vapuydenko@gmail.com. 